\title{
Measurement of mean cardiac dose for various breast irradiation techniques and corresponding risk of major cardiovascular event
}

\author{
Tomas Rodrigo Merino Lara ${ }^{1,2,3}$, Emmanuelle Fleury ${ }^{2,3}$, Shahram Mashouf ${ }^{2,3}$, Joelle Helou ${ }^{2,3}$, \\ Claire McCann 2,3, Mark Ruschin ${ }^{2,3}$, Anthony Kim ${ }^{2,3}$, Nadiya Makhani ${ }^{2,3}$, Ananth Ravi ${ }^{2,3}$ and \\ Jean-Philippe Pignol ${ }^{3,4}$ *
}

${ }^{1}$ Radiotherapy Unit, School of Medicine, Pontificia Universidad Católica de Chile, Santiago, Chile

${ }^{2}$ Department of Radiation Oncology, Sunnybrook Odette Cancer Centre, Toronto, ON, Canada

${ }^{3}$ Department of Radiation Oncology, University of Toronto, Toronto, ON, Canada

${ }^{4}$ Department of Radiation Oncology, Erasmus MC, Rotterdam, Netherlands

Edited by:

Bruce George Haffty, Rutgers Cancer Institute of New Jersey, USA

Reviewed by:

Matthew T. Studenski, University of Miami, USA

Volodymyr Nahirnyak, Bukovina State Medical University, Ukraine

*Correspondence:

Jean-Philippe Pignol, Department of

Radiation Oncology, Erasmus

MC - Daniel den Hoed, Groene

Hilledijk 301, Rotterdam 3075 EA,

Netherlands

e-mail: j.p.pignol@erasmusmc.nl
After breast conserving surgery, early stage breast cancer patients are currently treated with a wide range of radiation techniques including whole breast irradiation (WBI), accelerated partial breast irradiation (APBI) using high-dose rate (HDR) brachytherapy, or 3Dconformal radiotherapy (3D-CRT). This study compares the mean heart's doses for a left breast irradiated with different breast techniques. An anthropomorphic Rando phantom was modified with gelatin-based breast of different sizes and tumors located medially or laterally. The breasts were treated with WBI, 3D-CRT, or HDR APBI. The heart's mean doses were measured with Gafchromic films and controlled with optically stimulated luminescent dosimeters. Following the model reported by Darby (1), major cardiac were estimated assuming a linear risk increase with the mean dose to the heart of $7.4 \%$ per gray. WBI lead to the highest mean heart dose $(2.99 \mathrm{~Gy})$ compared to 3D-CRT APBI $(0.51 \mathrm{~Gy})$, multicatheter $(1.58 \mathrm{~Gy})$, and balloon HDR $(2.17 \mathrm{~Gy})$ for a medially located tumor. This translated into long-term coronary event increases of $22,3.8,11.7$, and $16 \%$ respectively. The sensitivity analysis showed that the tumor location had almost no effect on the mean heart dose for 3D-CRT APBI and a minimal impact for HDR APBI. In case of WBI large breast size and set-up errors lead to sharp increases of the mean heart dose. Its value reached $10.79 \mathrm{~Gy}$ for women with large breast and a set-up error of $1.5 \mathrm{~cm}$. Such a high value could increase the risk of having long-term coronary events by $80 \%$. Comparison among different irradiation techniques demonstrates that 3D-CRT APBI appears to be the safest one with less probability of having cardiovascular events in the future. A sensitivity analysis showed that WBI is the most challenging technique for patients with large breasts or when significant set-up errors are anticipated. In those cases, additional heart shielding techniques are required.

Keywords: breast neoplasms, radiotherapy, heart diseases, brachytherapy, radiation dosage

\section{INTRODUCTION}

In developed countries, breast cancer is the most common type of cancer in women $(2,3)$. With implementation of mammographic screening, the majority of the cases are diagnosed at an early stage. The standard treatment for early stage breast cancer includes removing the tumor and sampling the axillary lymph nodes using limited surgery (4). This is followed by whole breast radiotherapy and possibly regional radiation if nodes are positive. Currently, there is a general trend toward treatment de-escalation. Radiation oncology studies demonstrate that the duration of whole breast irradiation (WBI) can be shortened from 6 to 3 weeks (5-7), and other showing that for selected cases the amount of irradiated breast tissue could be limited to a small portion surrounding the surgical cavity $(8,9)$. This leads to a technique called accelerated partial breast irradiation (APBI). It combines a reduction of the irradiated breast volume and delivery of higher dose per fraction.
Multiple APBI techniques have been proposed including external beam 3D-conformal radiotherapy (3D-CRT), high-dose rate (HDR) interstitial brachytherapy using multicatheter or balloon, and permanent breast seeds implants $(10,11)$. As a result, patients with early stage breast cancers are treated with a variety of radiation techniques that appears comparable in terms of effectiveness $(12,13)$.

Along with the changes in radiation oncology practice mentioned above, the increased early detection of breast cancer due to screening programs has also resulted into improvement of the breast cancer treatment outcomes, with specific survival rates of $98.6 \%$ at 5 years (14). With improved survival, the reduction of treatment induced morbidity and mortality has gained importance as they may eliminate the need for adjuvant radiotherapy. Several studies with long-term follow-up have shown that standard external beam radiotherapy can increase the risk of ischemic heart 
disease and a recent large case control study suggests that a doseresponse relationship between the mean dose to the heart and the long-term risk of major cardiovascular events including mortality $(1,15-18)$. It is unknown if all the radiation techniques used in early stage breast cancer have similar cardiac risks since there are no long-term prospective data comparing them on this specific outcome. There is a limited number of studies reporting or comparing the heart dose (19-25) for one or two techniques but there has not been thorough comparison of the mean dose to the heart for all breast techniques, for various breast sizes and/or seroma locations. In most of cases, commercial treatment planning systems (TPS) are used for estimation of the heart's dose. Since this dose is calculated outside the field where photon scattering dominates, some concern about the accuracy of those calculations exists $(26,27)$.

Given the inaccuracies in calculating out of field dose with the current clinical TPS, the purpose of this study was to measure and compare the mean heart dose for different breast irradiation techniques delivered to the left breast of an anthropomorphic phantom. In addition, the robustness of our findings was tested using a sensitivity analysis looking at the added influence of breast size, seroma location, and organ motion.

\section{MATERIALS AND METHODS \\ PREPARATION OF PHANTOMS}

An anthropomorphic Rando phantom (The Phantom Laboratory, Salem, NY, USA) was modified using molded pieces of a tissue equivalent gel for mimicking various breast sizes. In order to prepare the necessary phantom, three CT scans of patients with left sided breast cancer having typically small $(300 \mathrm{cc})$, medium $(800 \mathrm{cc})$, and large breast $(1200 \mathrm{cc})$ volumes were selected from our institution's dosimetry database. Each CT slice was spaced by $1 \mathrm{~cm}$ and was printed on a scale $1: 1$ and used to create a realistic 3D breast shape assembling several styrofoam sheets of $1 \mathrm{~cm}$ thickness. The printout of the patient contour was pasted on individual styrofoam sheets and cut following the chest wall and breast contours (Figure 1). A negative breast mold was then made using a thermoplastic sheet. This negative mold was then filled with a tissue equivalent powdered ballistics gelatin (Vyse, Schiller Park, IL, USA) dissolved in water. The breast phantom was refrigerated overnight. The resulting gelatin phantom has an average CT number of 24 Hounsfield units (HU), which is similar to fibroglandular breast tissue. The phantom was kept at $5^{\circ} \mathrm{C}$ to limit melting and water evaporation. It was tightly fixed on the Rando phantom chest wall for planning and treatment.

\section{TREATMENT SIMULATION AND VOLUME DEFINITION}

Treatment simulation for small, medium, and large breasts was done following standard institution protocol (28). The Rando phantom with various breast volumes placed on the torso was positioned on a breast board. Five radio-opaque beads and/or pen marks were placed on the skin in the lateral, medial, inferior, and superior aspects of the chest to ensure treatment reproducibility. CT slices of $5 \mathrm{~mm}$ spacing and $5 \mathrm{~mm}$ thickness were acquired with a Philips CT scanner (Philips Healthcare, Andover, MA, USA) and transferred to either the Pinnacle 3 (RaySearch Americas Inc., Garden City, NY) or the Oncentra Brachytherapy planning systems (Nucletron Elekta, Stockholm, Sweden).

For WBI the clinical target volume (CTV) was defined as whole breast gel phantom limited by the Rando chest wall and a $5 \mathrm{~mm}$ layer below the phantom surface. For APBI, the CTV were defined either on the medial or the lateral quadrants of the breast. To ensure comparison of similar target volumes, CTVs of $60 \mathrm{cc}$ were delineated. For brachytherapy, the planning target volume (PTV) included a $1.5 \mathrm{~cm}$ expansion from the CTV but limited to the Rando chest wall and $5 \mathrm{~mm}$ below the breast surface, while for 3D-CRT APBI the PTV included an expansion of $2.5 \mathrm{~cm}$, similarly to the NSABP-B39 protocol (29).

\section{TREATMENT PROTOCOLS}

\section{External beam radiotherapy}

Whole breast irradiation was planned following standard breast IMRT protocol (30) using a prescription dose of $50 \mathrm{~Gy}$ in

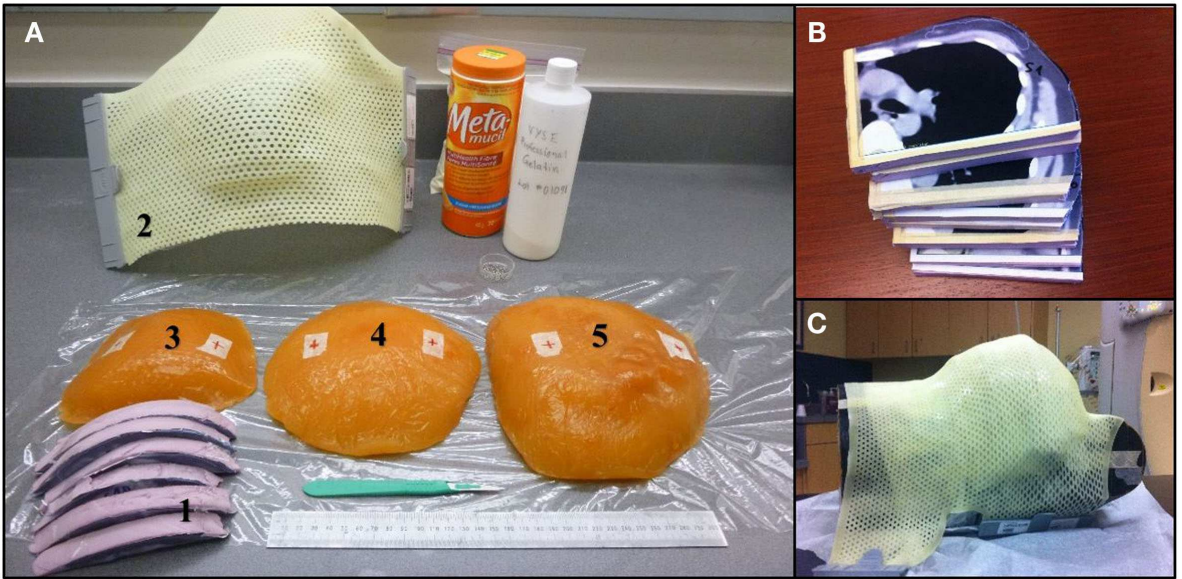

FIGURE 1 | (A) Materials used during the breast phantom manufacture. (1) Styrofoam slices cut to fit CT contours. (2) Thermoplastic 3D breast contour obtained from the Styrofoam mold. (3-5) Small, medium, and large size of gelatin-based breast phantoms. (B) Styrofoam slices cut to the patient profile using CT images. (C) Thermoplastic mold over the Rando phantom modified with the large breast to ensure good contact. 


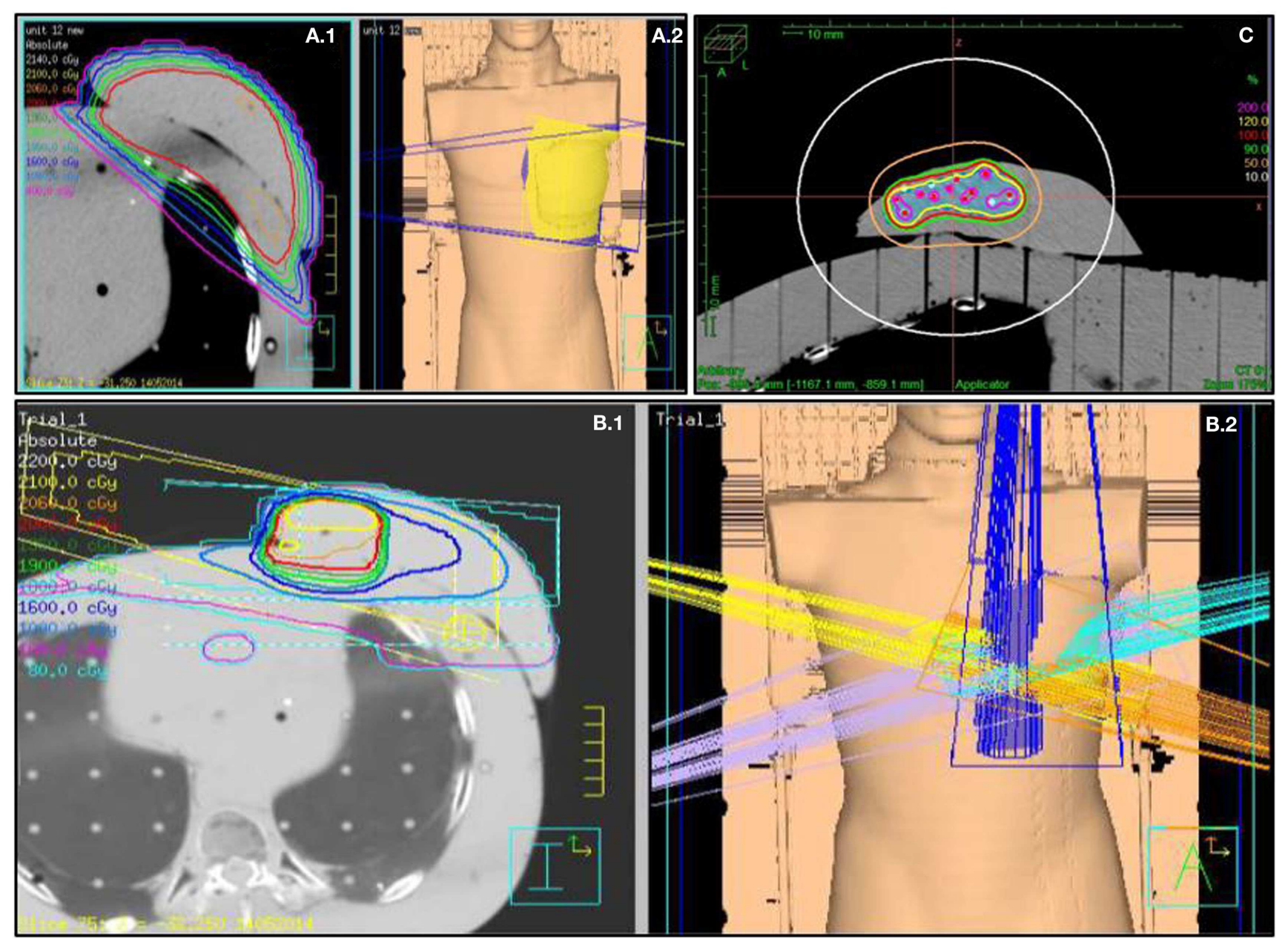

FIGURE 2 | Fields arrangement and various breast treatments dosimetry. (A.1) Medium size WBI treatment dosimetry and (A.2) tangential fields 3D representation. (B.1) 3D-CRT APBI dosimetry and (B.2) five fields no coplanar 3D representation. (C) Multicatheter HDR APBI dosimetry.

25 fractions. For the small and the medium-sized breast phantoms, the beam energy was $6 \mathrm{MV}$, while a mix of 6 and $18 \mathrm{MV}$ beams was used for the large breast volume. In this protocol, a multileaf collimator (MLC) is used to shape several field-in-field beams to compensate for missing tissue and to improve the dose distribution homogeneity. Plans were normalized to a prescription point set at mid-separation, 2/3 of the distance between skin and a base of the tangential fields. Heart shielding involved ensuring the anterior heart volume was away from the posterior beam edge. Standard treatment set-up procedures were followed including verification of each field using portal imaging.

For 3D-CRT APBI, three to five non-coplanar beams were aimed (Figure 2$)$ at the PTV $(29,31)$ and a dose of $38.5 \mathrm{~Gy}$ in 10 BID fractions was prescribed. The distribution was normalized on the PTV centroid.

Treatments were delivered using a 6/18 MV Elekta Synergy linac equipped with a multileave collimator (Elekta Inc., Crawley, UK). Treatment was delivered after verification of the correctness of the set-up using portal imaging.

\section{Brachytherapy}

Using a free-hand technique, 13 catheters were inserted (Figure 3 ) in a triangular pattern and evenly spaced by $1.5 \mathrm{~cm}$ in the horizontal plane and $1 \mathrm{~cm}$ in the vertical plane (32). The implanted Rando phantom was CT simulated and the images were transferred to the planning system for target segmentation and dose optimization. A dose of $34 \mathrm{~Gy}$ in 10 fractions was prescribed on the minimal peripheral dose (MPD) and dwell times were optimized using the IPSA optimization module (33) to ensure that at least $90 \%$ of the target volume $\left(\mathrm{D}_{90}\right)$ will receive at least $90 \%$ of the prescribed dose, and that the volume receiving more that $200 \%$ of the prescribed dose $\left(\mathrm{V}_{200}\right)$ would be $<20 \mathrm{cc}$. HDR brachytherapy was delivered using a ${ }^{192}$ Ir HDR remote afterloader (Flexitron, Elekta, Stockholm, Sweden). To replicate a balloon catheter HDR treatment, a $3 \mathrm{~cm}$ diameter surgical cavity was made in the breast gel phantom and a Foley catheter was positioned inside before being filled with saline. A single catheter was inserted into the Foley catheter and used to deliver a dose of $34 \mathrm{~Gy}$ in 10 fractions at the point located $1 \mathrm{~cm}$ from the balloon surface. 


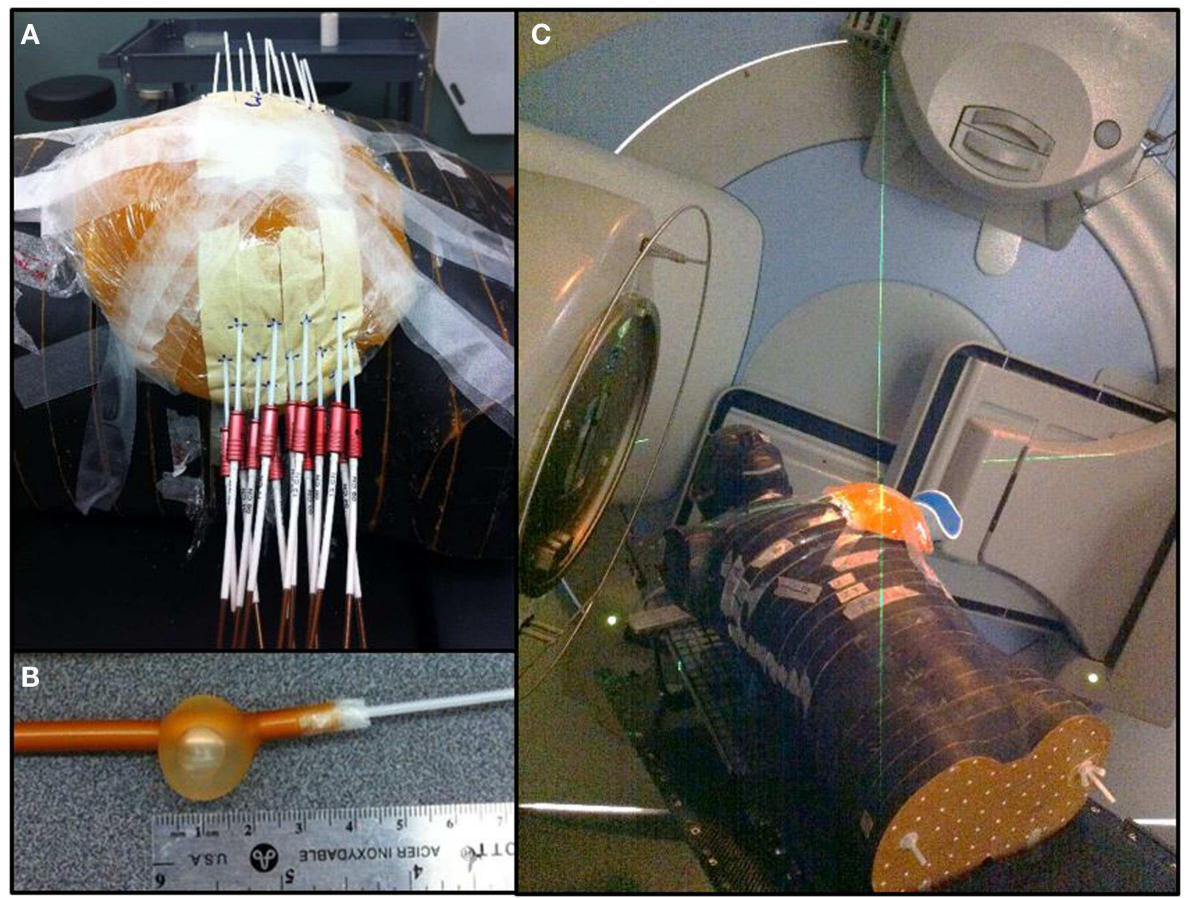

FIGURE 3 | Breast treatments. (A) Multicatheter HDR APBI. (B) Foley catheter used for balloon HDR APBI. (C) 3D-CRT APBI.

\section{PLACEMENT OF DOSIMETERS}

Two types of dosimeters were used for dose measurements, optically stimulated luminescent dosimeter (OSLD) high-accuracy Nanodot dosimeters (Landauer Inc., Glenwood, IL, USA) and Gafchromic EBT3 films (Ashland Inc., Covington, KY, USA). OSLD dosimeters were placed in areas corresponding to a left descending artery, and the center of left and right ventricles. The detectors were placed inside a bolus material between the three consecutive Rando phantom slices where the heart was identified (Figure 4).

Three Gafchromic films were used to evaluate the heart's dose distribution in $3 \mathrm{D}$. They were positioned at different location evenly spaced by $1.5 \mathrm{~cm}$. In total, 24 films were irradiated at the three films positions (Apex, medium heart, and base of the heart). For the each technique, films were placed between slices inside the anthropomorphic phantom and fixed in clearly established positions for every experiment. To indicate the exact position of the contour of the heart, the heart contour was drawn on the film with a permanent marker.

In accordance with recommendations of AAPM TG55, the Gafchromic films were kept in a dry and dark area at room temperature for at least $24 \mathrm{~h}$ before reading. The heart contours identified on the films were segmented and the optical density was found using the Epson Expression 10000XL scanner (EPSON Deutschland $\mathrm{GmbH}$, Meerbusch, Germany). Optical densities were converted into dose using a calibration curve. All measured dose were expressed as a percentage of the prescribed dose. The doses measured with the $2 \mathrm{D}$ film were assumed to represent the average dose absorbed in the adjacent heart's volume and cumulative DVH were built.

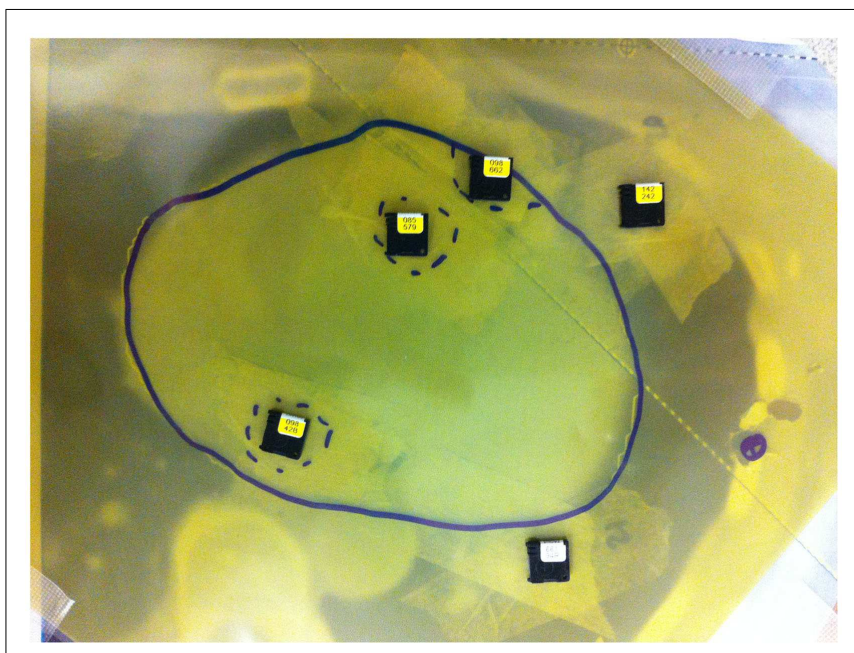

FIGURE 4 | Optically stimulated luminescent dosimeters and Gafchromic film placement between Rando slices with a $5 \mathrm{~mm}$ bolus

\section{SENSITIVITY ANALYSIS}

Except the used radiation techniques, other changing factors such as the breast size, shape, and location of the seroma, and distance between a heart and a field's border or a HDR source could also impact on the mean heart dose (34). A meaningful evaluation of the mean heart dose should also account for potential patient set-up error, also called inter-fraction error, for anatomical factors such as heart volume variations between the systolic and diastolic phases or due to patient's phenotype. To evaluate the impact of 
those variations for various radiation techniques, the Gafchromic films were reanalyzed shifting the heart position by $1.5 \mathrm{~cm}$. This value is the average of the distance between the field border and the tip of the heart measured on portal imaging for the worse case scenario group in Goody's study (35). In this report, $11 \%$ of the 128 patients had the heart protruding in the irradiation field from 10 to $20 \mathrm{~mm}$.

\section{ESTIMATION OF MAJOR CARDIAC EVENTS}

Following the model reported by Darby (1), major cardiac were estimated assuming a linear risk increase with the mean dose to the heart of $7.4 \%$ per gray (95\% confidence interval, 2.9-14.5; $p<0.001)$. Those major cardiac events include myocardial infarction, coronary revascularization, and death from ischemic heart disease, but angina episodes are not included.

\section{RESULTS}

\section{QUALITY ASSURANCE}

The OSLD dose measurements performed inside breast of various sizes were in very good agreement with those calculated with Pinnacle TPS. The dose measured using three to five detectors placed inside the breast was $95 \%(\mathrm{SD}=2.5 \%)$ of the calculated one for the small breast, and $101 \%(\mathrm{SD}=0.8 \%)$ of the calculated one for the medium size breast.

A very good agreement between OSLD measurements and the Gafchromic film measurements were received (Figure 5). A correlation coefficient $R^{2}$ of $0.98(p<0.001)$ is calculated.

\section{MEAN DOSE TO THE HEART}

The measured mean heart's doses received with different irradiation technique for medium size breast are shown in Table 1. WBI

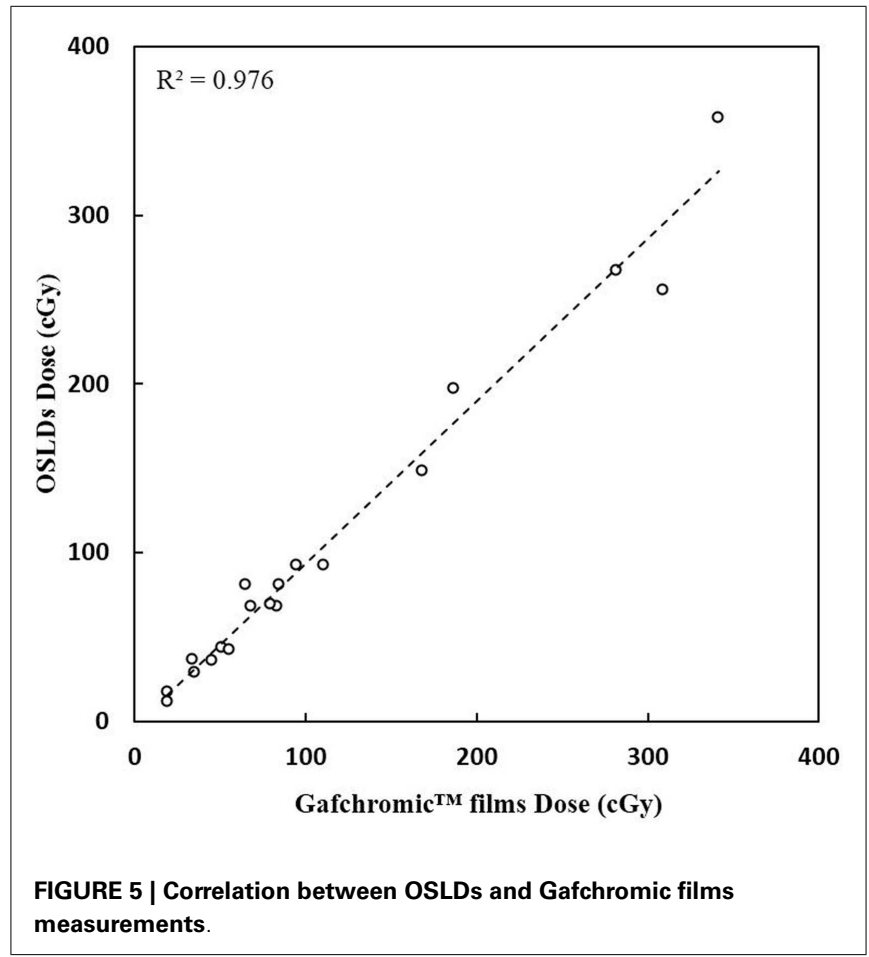

yielded the highest mean heart dose, 2.9 Gy, leading to an estimated increased risk of major coronary events of $22 \%$, while the lowest mean heart dose was measured for the 3D-CRT APBI, $0.5 \mathrm{~Gy}$, leading to a negligible $4 \%$ increased risk of cardiac events. The summarized cumulative DVHs for different radiation techniques and different anatomical structures are shown on Figures 6-9.

\section{SENSITIVITY ANALYSIS}

We performed a sensitivity analysis. There was no significant difference in the value of the mean heart's dose when the small and medium breast size phantoms were used. At the same time, its value doubled from 2.99 Gy to $6.39 \mathrm{~Gy}$ when the largest breast size phantom was used (Table 2). This was due to the posterior shift of the beam edge needed to fully cover the breast volume. The seroma location had little impact when using whole breast radiotherapy or 3D-CRT APBI. It increased the mean heart's dose by $17 \%$ for a medially compared to laterally located seroma using multicatheter brachytherapy, and by $32 \%$ using balloon brachytherapy. This was essentially due to the closer proximity to the radioactive source. The most dramatic increase of mean heart's dose was seen when we were testing set-up or organ motion errors for external beam radiotherapy. An anterior shift of the heart's edge by $1.5 \mathrm{~cm}$ resulted in a $150 \%$ increase. For a large breast volume, the mean heart dose could reach $10.8 \mathrm{~Gy}$, which corresponds to almost a twofolds increased risk of major coronary events. The set-up errors and organ motion effects were much less pronounced for brachytherapy techniques and this was the consequence of the smoother isodose gradient on the Gafchromic films anteriorly to the heart compared to external beam with heart shielding.

Table 1 | Mean heart dose measured with Gafchromic films for the medium $(800 \mathrm{cc})$ and large breast $(1200 \mathrm{cc})$ phantom using different radiation techniques.

\begin{tabular}{|c|c|c|c|}
\hline Technique & $\begin{array}{l}\text { Mean } \\
\text { dose (Gy) }\end{array}$ & $\begin{array}{l}\text { Relative to } \\
\text { prescribed } \\
\text { dose }(\%)\end{array}$ & $\begin{array}{l}\text { Increased risk } \\
\text { of coronary events } \\
\text { in } \%(95 \% \mathrm{Cl})^{b}\end{array}$ \\
\hline \multicolumn{4}{|l|}{ WBI } \\
\hline Medium (800 cc) & 2.99 & 5.99 & $22.0(8.7-43.4)$ \\
\hline Large $(1200 \mathrm{cc})^{\mathrm{a}}$ & 6.39 & 12.79 & 47.2 (18.5-92.6) \\
\hline \multicolumn{4}{|l|}{ 3D-CRT-APBI } \\
\hline Lateral & 0.57 & 1.48 & $4.2(1.7-8.3)$ \\
\hline Medial & 0.51 & 1.34 & $3.8(1.5-7.4)$ \\
\hline \multicolumn{4}{|c|}{ HDR MULTICATHETER } \\
\hline Lateral & 1.44 & 4.28 & 10.6 (4.2-20.9) \\
\hline Medial & 1.58 & 4.67 & 11.7 (4.6-22.9) \\
\hline \multicolumn{4}{|l|}{ HDR BALLOON } \\
\hline Lateral & 1.27 & 3.73 & $9.4(3.7-18.4)$ \\
\hline Medial & 2.17 & 6.38 & $16.0(6.3-31.5)$ \\
\hline
\end{tabular}

a Large pendular breast treated wide tangents.

b/ncreased risk in major coronary events (myocardial infarction, coronary revascularization, and death from ischemic heart disease) is $7.4 \%$ (95\% confidence interval 2.9-14.5\%) per Gray (16).

WBI, whole breast irradiation; 3D-CRT APBI, 3D-conformal radiation therapy accelerated partial breast irradiation 


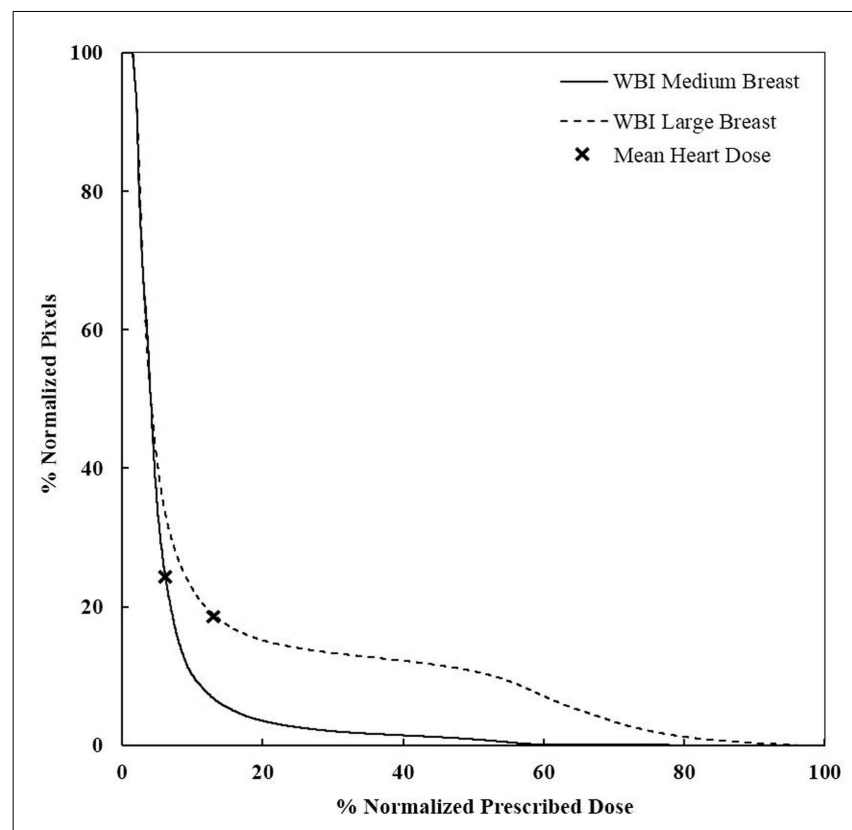

FIGURE 6 | DVHs for WBI of medium and large breasts. More heart is receiving a higher dose for large breasts. WBI, whole breast irradiation.

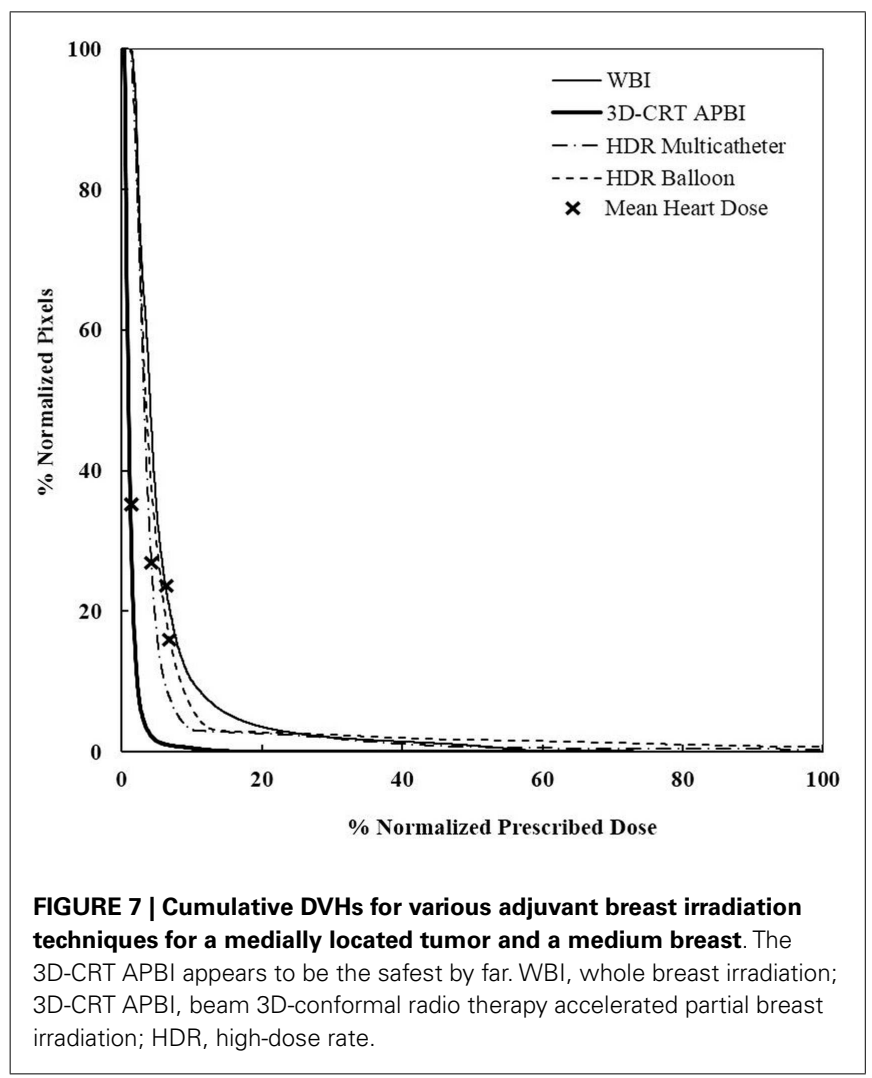

\section{DISCUSSION}

This work reports the mean cardiac doses measured in an anthropometric phantom mimicking, a patient receiving breast radiotherapy with various techniques currently used for early

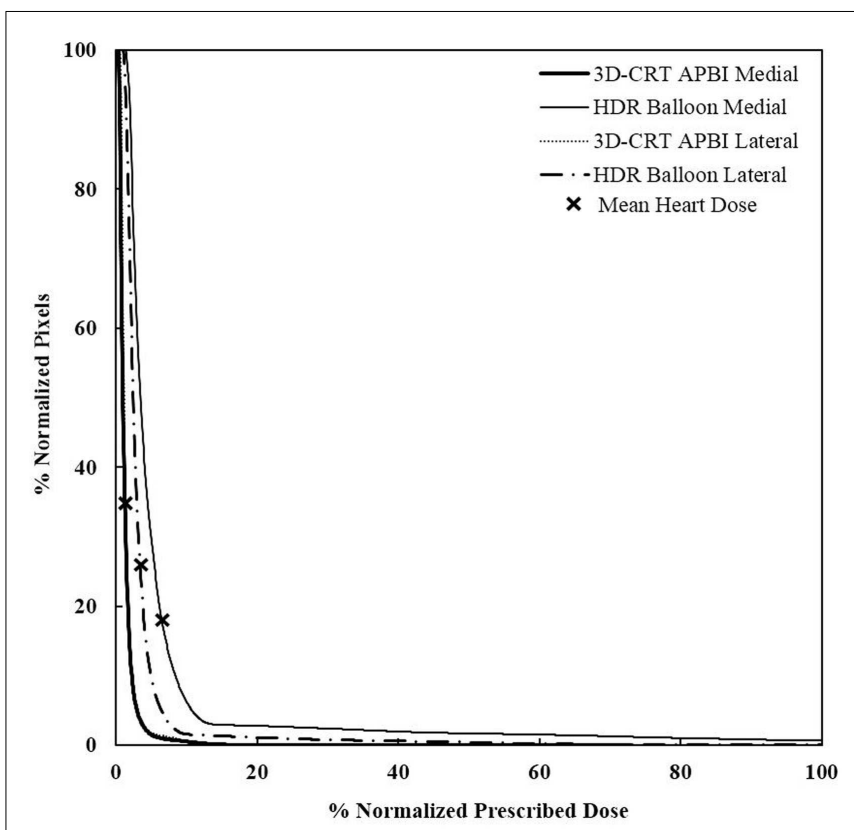

FIGURE 8 | Cumulative DVHs for various tumor locations and APBI techniques. There is no impact of location for 3D-CRT as opposed to HDR techniques. 3D-CRT APBI, beam 3D-conformal radio therapy accelerated partial breast irradiation; HDR, high-dose rate.

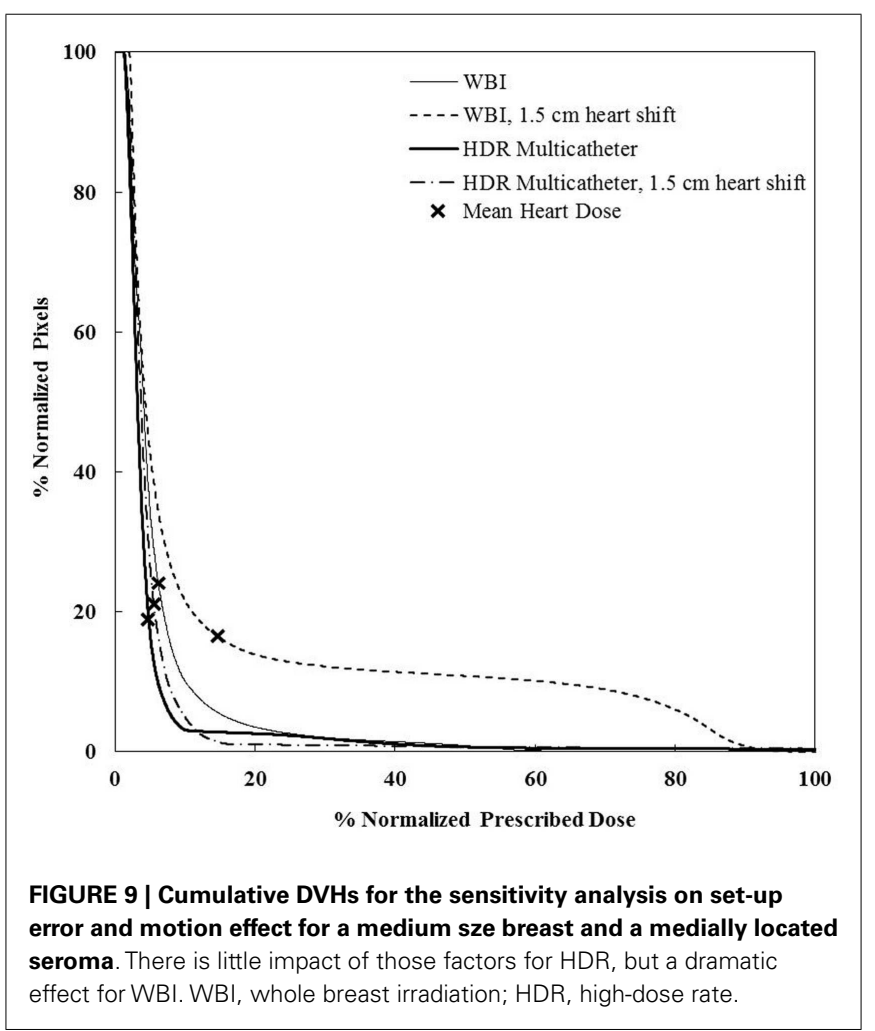

stage breast cancer treatment. This study provides experimental data that could be considered more reliable compared to those calculated in commercial TPS. According to other publications, 
Table 2 | Set-up error and organ motion sensitivity analysis of the mean heart dose for the medium $(800 \mathrm{cc})$ and large breast phantom (1200 cc) using a $1.5 \mathrm{~cm}$ anterior heart shift

\begin{tabular}{|c|c|c|c|}
\hline Technique & $\begin{array}{l}\text { Mean } \\
\text { dose (Gy) }\end{array}$ & $\begin{array}{l}\text { Relative to } \\
\text { prescribed } \\
\text { dose }(\%)\end{array}$ & $\begin{array}{l}\text { Increased risk of } \\
\text { coronary events } \\
\text { in } \%(95 \% \mathrm{Cl})\end{array}$ \\
\hline \multicolumn{4}{|l|}{ WBI } \\
\hline Medium (800 cc) & 7.11 & 14.22 & $52.6(20.6-100)$ \\
\hline Large $(1200 \mathrm{cc})^{\mathrm{a}}$ & 10.79 & 21.59 & $79.8(31.3-100)$ \\
\hline \multicolumn{4}{|l|}{ 3D-CRT-APBI } \\
\hline Lateral & 0.69 & 1.81 & $5.1(2.0-10.0)$ \\
\hline Medial & 1.20 & 3.14 & $8.9(3.5-17.4)$ \\
\hline \multicolumn{4}{|c|}{ HDR MULTICATHETER } \\
\hline Lateral & 1.68 & 4.97 & $12.4(4.9-24.4)$ \\
\hline Medial & 1.70 & 5.00 & $12.6(4.9-24.7)$ \\
\hline \multicolumn{4}{|l|}{ HDR BALLOON } \\
\hline Lateral & 1.34 & 3.96 & $9.9(3.9-19.4)$ \\
\hline Medial & 2.44 & 7.19 & $18.1(7.1-35.4)$ \\
\hline
\end{tabular}

a Large pendulous breast treated wide tangents.

WBI, Whole breast irradiation; 3D-CRT APBI, 3D-conformal radiation therapy accelerated partial breast irradiation.

commercial TPS significantly underestimate the scatter dose outside the irradiation field $(27,35)$. Howell previously reported that the Eclipse's analytic anisotropic algorithm gave a dose at the point $11.25 \mathrm{~cm}$ away from the treatment field border less by $55 \%$ than that of measured directly with thermoluminescent dosimeters (TLD) (35). To address this issue, our group used Monte Carlo simulation to estimate the dose delivered to the left anterior descending artery in an anthropometric phantom. Because secondary photons rarely crossed the volume of interest (VOI) the transport of a very large number of photons and multiple variance reduction strategies were necessary. Major simplifications have been made to the description of the phantom including large tally volumes (36).

Yet, since very low values are expected, the measurement of scattered dose remains challenging. The following quality assurance measures were undertaken to control the validity of our measurement. First, the doses at several points were checked using two independent methods, namely, the OSLDs and the Gafchromic films. Both are energy independent and the second one enables capturing a 3D spatial dose distribution stacking films. Second, we compared doses measured inside the high-dose treated volume and those calculated by the TPS. Those checks were considered satisfactory if they show differences in dose lower than 5\%. Third, we repeated the experiments two times to ensure no major set-up error was made.

The most noteworthy finding of our study is that the mean heart dose was almost halved when using HDR APBI compared to WBI, even for the worst case scenario of a medially located left breast tumor. In this instance, the balloon brachytherapy technique does slightly worse, but still better than whole breast radiotherapy. The use of 3D-CRT APBI reduced the mean heart dose to one-third of what is received with use of HDR and to a sixth compared to WBI. This was essentially due to the limited extension of the posterior field border compared to WBI. It turns out the 3DCRT APBI is the safest radiation technique and its use has the lowest risk of having major cardiovascular events. Those findings are consistent with previously reported ones. In a dose modeling study, Hiatt reported a sixfolds dose reduction when 3D-CRT was used instead of whole breast using IMRT (37). Also, Valach (25) reported a mean heart's dose of $2.45 \pm 0.94$ Gy when balloon brachytherapy was used and the seroma was located in the inner quadrant of the left breast. Our measured value for a similar case was equal to $2.17 \mathrm{~Gy}$.

One limitation of the present study is linked to the use of the Rando phantom that imposes estimating the 3D mean heart's dose using only three Gafchromic films. Since the dose gradients are very smooth for the APBI technique, any impact would mainly concern the WBI technique where steep gradients are seen in the portion of the heart close to the beam edge. However, the films were placed perpendicular to the beam direction, such that a fine dose resolution was obtained in 2D. It is hence unlikely that any cold or hot spot may have been missed and that additional resolution would significantly change our findings. In addition, the main goal of the present study is not to provide exact value of mean heart's dose since they could vary depending on many factors. The purpose of this study was mainly to compare different breast techniques, so using the same methodology enable a fair comparison among them. Another limitation of our work relates to the conversion of mean heart's dose to major cardiovascular event risks for each radiation technique (18). The isodose fall-off in the heart is very different between WBI, HDR, and 3D-CRT APBI. It is much steeper for the first one and gradual for the other ones. This leads to very different DVH profiles and it is eventually unclear if comparing the mean instead of, for example, the median heart's dose is the right approach. Identifying the critical structures involved in the radiation damage to the heart remains challenging. Coronary arteries including the left anterior descending artery or ventricles have been suggested (38). There is, however, no data correlating the doses received on those volumes to a prospectively evaluated clinical endpoint. We used the model proposed by Darby, as it remains the only one showing a statistically significant correlation between a risk of major cardiac events and a dosimetry parameter. But, we acknowledge that the risk we calculated for the various breast techniques maybe over or underestimated.

Using the predictive model proposed by Darby (18), a large variation in the values of the major coronary event risk is obtained. It ranges from a negligible $4-5 \%$ increase, in case of $3 \mathrm{D}$-CRT and a medially or laterally located tumor, to a concerning $80 \%$ increase in case of a patient with a large breast having a systematic set-up error and/or motion exceeding $1.5 \mathrm{~cm}$. This emphasizes the need of individual evaluation of risks accounting for potential intra-fraction errors and for patients with a large size breast with risk of set-up error measures to reduce the dose delivered to the heart must be taken. Those measures include gating the radiation delivery to the breathing cycle, using a prone position, 3D-CRT APBI technique, or proton therapy (39-41). Techniques like moderate deep inspiration breath hold have now been widely introduced into clinic. Although there is no long-term data to confirm its benefit in term of major cardiac event reduction, long-term experience shows that the mean heart's dose is reduced by $40 \%$ (40). 
It must be noted that although the finding of a better cardiac shielding using APBI is clearly appealing for a cancer population with excellent survival rates, the long-term outcomes of APBI remains unknown. If the early outcomes from large trials and multiple cohort studies appear promising $(12,13,42,43)$, a large population-based study shows contrariwise a marginal increased rate of mastectomy likely linked to local recurrence (44). It is eventually difficult to evaluate the final impact on the overall survival when balancing those opposite effects.

\section{REFERENCES}

1. Darby SC, Ewertz M, McGale P, Bennet AM, Blom-Goldman U, Bronum D, et al. Risk of ischemic heart disease in women after radiotherapy for breast cancer. $N$ Engl J Med (2013) 368:987-98. doi:10.1056/NEJMoa1209825

2. DeSantis C, Ma J, Bryan L, Jemal A. Breast cancer statistics, 2013. CA Cancer J Clin (2014) 64:52-52. doi:10.3322/caac.21203

3. Youlden DR, Cramb SM, Dunn NA, Muller JM, Pyke CM, Baade PD. The descriptive epidemiology of female breast cancer: an international comparison of screening, incidence, survival and mortality. Cancer Epidemiol (2012) 36:237-48. doi:10.1016/j.canep.2012.02.007

4. Gradishar WJ, Anderson BO, Blair SL, Burstein HJ, Cyr A, Elias AD, et al. Breast cancer version 3.214. J Natl Compr Canc Netw (2014) 12:542-90.

5. START Trialist Group; Betzen SM, Agrawal RK, Air EG, Barret JM, Barret-Lee PJ, et al. The UK standardisation of breast radiotherapy (START) trial A of radiotherapy hypofractionation for the treatment of early breast cancer: a randomized trial. Lancet Oncol (2008) 9:331-41. doi:10.1016/S1470-2045(08)70077-9

6. START Trialist Group; Betzen SM, Agrawal RK, Air EG, Barret JM, Barret-Lee PJ, et al. The UK standardisation of breast radiotherapy (START) trial B of radiotherapy hypofractionation for the treatment of early breast cancer: a randomized trial. Lancet Oncol (2008) 371:1098-107. doi:10.1016/S0140-6736(08)60348-7

7. Whelan TJ, Pignol JP, Levine MN, Julian JA, Mackenzie R, Parpia S, et al. Longterm results of hypofractionated radiation therapy for breast cancer. $N$ Engl J Med (2010) 362:513-20. doi:10.1056/NEJMoa0906260

8. Smith BD, Arthur DW, Buchholtz TA, Haffty BG, Hahn CA, Hardenbergh PH, et al. Accelerated partial breast irradiation consensus statement from the American society for radiation oncology (ASTRO). Int J Radiat Oncol Biol Phys (2009) 74:987-1001. doi:10.1016/j.ijrobp.2009.02.031

9. Polgar C, Van Limbergen E, Potter R, Kovacs G, Polo A, Lyczek J, et al. Patient selection for accelerated partial-breast irradiation (APBI) after breastconserving surgery: recommendations of the groupe Européen de curiethérapie - European society for therapeutic radiology and oncology (GECESTRO) breast cancer working group based on clinical evidence. Radiother Oncol (2010) 94:264-73. doi:10.1016/j.radonc.2010.01.014

10. Njeh CF, Saunders M, Langton C. Accelerated partial breast irradiation (APBI): a review of available techniques. Radiat Oncol (2010) 5:90. doi:10.1186/1748717X-5-90

11. Pignol JP, Rakovitch E, Keller BM, Sankreacha R, Chartier C. Tolerance and acceptance results of a palladium-103 permanent breast seed implant phase I/II study. Int J Radiat Oncol Biol Phys (2009) 73:1482-8. doi:10.1016/j.ijrobp.2008. 06.1945

12. Shah C, Badiyan S, Ben Wilkinson J, Vicini F, Beitsch P, Keisch M, et al. Treatment efficacy with accelerated partial breast irradiation (APBI): final analysis of the American society of breast surgeons mammosite breast brachytherapy registry trial. Ann Surg Oncol (2013) 20:3279-85. doi:10.1245/s10434-013-3158-4

13. Vaidya JS, Wenz F, Bulsara M, Tobias JS, Joseph DJ, Keshtgar M, et al. Riskadapted targeted intraoperative radiotherapy versus whole-breast radiotherapy for breast cancer: 5-year results for local control and overall survival from the TARGIT-A randomised trial. Lancet (2014) 383:603-13. doi:10.1016/S01406736(13)61950-9

14. Howlader N, Noone AM, Krapcho M, Garshell J, Miller D, Altekruse SF, et al. SEER Cancer Statistics Review, 1975-2011. Bethesda, MD: National Cancer Institute (2014). Available from: http://seer.cancer.gov/csr/1975_2011/

15. Cuzick J, Stewart H, Rutqvist L, Houghton J, Edwards R, Redmond C, et al. Cause-specific mortality in long-term survivors of breast cancer who participated in trials of radiotherapy. J Clin Oncol (1994) 12:447-53.

16. Tjessem KH, Johansen S, Malinen E, Reinertsen KV, Danielsen T, Fosså SD, et al. Long-term cardiac mortality after hypofractionated radiation therapy in breast cancer. Int J Radiat Oncol Biol Phys (2013) 87:337-43. doi:10.1016/j.ijrobp.2013. 05.038

17. Paszat LF, Mackillop WJ, Groome PA, Boyd C, Schulze K, Holowaty E. Mortality from myocardial infarction after adjuvant radiotherapy for breast cancer in the surveillance, epidemiology, and end-results cancer registries. J Clin Oncol (1998) 16:2625-31.

18. Darby SC, McGale P, Taylor CW, Peto R. Long-term mortality from heart disease and lung cancer after radiotherapy for early breast cancer: prospective cohort study of about 300,000 women in US SEER cancer registries. Lancet Oncol (2005) 6:557-65. doi:10.1016/S1470-2045(05)70251-5

19. Lettmaier S, Kreppner S, Lotter M, Walser M, Ott OJ, Fietkau R, et al. Radiation exposure of the heart, lung and skin by radiation therapy for breast cancer: a dosimetric comparison between partial breast irradiation using multicatheter brachytherapy and whole breast teletherapy. Radiother Oncol (2011) 100:189-94. doi:10.1016/j.radonc.2010.07.011

20. Garza R, Albuquerque K, Sethi A. Lung and cardiac tissue doses in left breast cancer patients treated with single-source breast brachytherapy compared to external beam tangent fields. Brachytherapy (2006) 5:235-8. doi:10.1016/j.brachy. 2006.08.001

21. Weed DW, Edmunson GK, Vicini FA, Chen PY, Martinez AA. Accelerated partial breast irradiation: a dosimetric comparison of three different techniques. Brachytherapy (2005) 4:121-9. doi:10.1016/j.brachy.2004.12.005

22. Major T, Niehoff P, Kovacs G, Fodor J, Polgar C. Dosimetric comparisons between high dose rate interstitial mamosite balloon brachytherapy for breast cancer. Radiother Oncol (2006) 79:321-8. doi:10.1016/j.radonc.2006.05.005

23. Khan AJ, Kirk MC, Mehta PS, Seif NS, Griem KL, Bernard DA, et al. A dosimetric comparison of three-dimensional conformal, intensity modulated radiation therapy, and mamosite partial-breast irradiation. Brachytherapy (2006) 5:183-8. doi:10.1016/j.brachy.2006.06.001

24. Stewart AJ, O'Farrell DA, Cormack RA, Hansen JL, Khan AJ, Mutyala S, et al. Dose volume histogram analysis of normal structures associated with accelerated partial breast irradiation delivered by high dose rate brachytherapy and comparison with whole breast external beam radiotherapy. Radiat Oncol (2008) 19:3-39. doi:10.1186/1748-717X-3-39

25. Valakh V, Kim Y, Werts ED, Trombetta MG. A comprehensive analysis of cardiac dose in balloon-based high-dose-rate brachytherapy for left-sided breast cancer. Int J Radiat Oncol Biol Phys (2012) 82:1698-705. doi:10.1016/j.ijrobp.2011.02. 058

26. Taylor ML, Kron T. Consideration of the radiation dose delivered away from the treatment field to patients in radiotherapy. Phys Med Biol (2011) 36:59-71. doi:10.4103/0971-6203.79686

27. Howell RM, Scarboro SB, Kry SF, Yaldo DZ. Accuracy of out-of-field dose calculations by a commercial treatment planning system. Phys Med Biol (2010) 55:6999-7008. doi:10.1088/0031-9155/55/23/S03

28. Woo TC, Pignol JP, Rakovitch E, Vu T, Hicks D, O’Brien P, et al. Body radiation exposure in breast cancer radiotherapy: impact of breast IMRT and virtual wedge compensation techniques. Int J Radiat Oncol Biol Phys (2006) 65:52-8. doi:10.1016/j.ijrobp.2005.11.023

29. NSABP B-39, RTOG 0413: a randomized phase III study of conventional whole breast irradiation versus partial breast irradiation for women with stage $0, \mathrm{I}$, or II breast cancer. Clin Adv Hematol Oncol (2006) 4:719-21.

30. Pignol JP, Olivotto I, Rakovitch E, Gardner S, Sixel K, Beckham W, et al. A multicenter randomized trial of breast intensity-modulated radiation therapy to reduce acute radiation dermatitis. J Clin Oncol (2008) 26:2085-92. doi:10.1200/JCO.2007.15.2488

31. Olivotto IA, Whelan TJ, Parpia S, Kim DH, Berrang T, Truong PT, et al. Interim cosmetic and toxicity results from RAPID: a randomized trial of accelerated partial breast irradiation using three-dimensional conformal external beam radiation therapy. J Clin Oncol (2013) 31:4038-45. doi:10.1200/JCO.2013.50.5511

32. Pierquin B, Dutreix A, Paine CH, Chassagne D, Marinello G, Ash D. The Paris system in interstitial radiation therapy. Acta Radiol Oncol Radiat Phys Biol (1978) 17:33-48. doi:10.3109/02841867809127689

33. Lessard E, Pouliot J. Inverse planning anatomy-based dose optimization for HDR-brachytherapy of the prostate using fast simulated annealing algorithm and dedicated objective function. Med Phys (2001) 28:773-9. doi:10.1118/1. 1368127

34. Taylor CW, McGale P, Povall JM, Thomas E, Kumar S, Dodwell D, et al. Estimating cardiac exposure from breast cancer radiotherapy in clinical practice. Int J Radiat Oncol Biol Phys (2009) 73:1061-8. doi:10.1016/j.ijrobp.2008.05.066 
35. Goody RB, O’Hare J, McKenna K, Dearey L, Robinson J, Bell P, et al. Unintended cardiac irradiation during left-sided breast cancer radiotherapy. Br J Radiol (2013) 86:20120434. doi:10.1259/bjr.20120434

36. Pignol JP, Keller BM, Ravi A. Doses to internal organs for various breast radiation techniques-implications on the risk of secondary cancers and cardiomyopathy. Radiat Oncol (2011) 6:5. doi:10.1186/1748-717X-6-5

37. Hiatt JR, Evans SB, Price LL, Cardarelli GA, Dipetrillo TA, Wazer DE. Dosemodeling study to compare external beam techniques from protocol NSABP B39/RTOG 0413 for patients with highly unfavorable cardiac anatomy. Int J Radiat Oncol Biol Phys (2006) 65(5):1368-74. doi:10.1016/j.ijrobp.2006.03.060

38. Correa CR, Litt HI, Hwang WT, Ferrari VA, Solin LJ, Harris EE. Coronary artery findings after left-sided compared with right-sided radiation treatment for earlystage breast cancer. J Clin Oncol (2007) 25:3031-7. doi:10.1200/JCO.2006.08 6595

39. Shah C, Badiyan S, Berry S, Khan AJ, Goyal S, Schulte K, et al. Cardiac dose sparing and avoidance techniques in breast cancer radiotherapy. Radiother Oncol (2014) 112(1):9-16. doi:10.1016/j.radonc.2014.04.009

40. Swanson T, Grills IS, Ye H, Entwistle A, Teahan M, Letts N, et al. Six-year experience routinely using moderate deep inspiration breath-hold for the reduction of cardiac dose in left-sided breast irradiation for patients with earlystage or locally advanced breast cancer. Am J Clin Oncol (2013) 36:24-30. doi:10.1097/COC.0b013e31823fe481

41. Jimenez RB, Goma C, Nyamwanda J, Kooy HM, Halabi T, Napolitano BN, et al. Intensity modulated proton therapy for postmastectomy radiation of bilateral implant reconstructed breasts: a treatment planning study. Radiother Oncol (2013) 107:213-7. doi:10.1016/j.radonc.2013.03.028

42. Zauls AJ, Watkins JM, Wahlquist AE, Brackett NC III, Aguero EG, Baker MK, et al. Outcomes in women treated with mammosite brachytherapy or whole breast irradiation stratified by ASTRO accelerated partial breast irradiation consensus statement groups. Int J Radiat Oncol Biol Phys (2012) 82:21-9. doi:10.1016/j.ijrobp.2010.08.034

43. Park SS, Grills IS, Chen PY, Kestin LL, Ghilezan MI, Wallace M, et al. Accelerated partial breast irradiation for pure ductal carcinoma in situ. Int J Radiat Oncol Biol Phys (2011) 81:403-8. doi:10.1016/j.ijrobp.2010.05.030

44. Smith GL, Xu Y, Buchholz TA, Giordano SH, Jiang J, Shih YC, et al. Association between treatment with brachytherapy vs whole-breast irradiation and subsequent mastectomy, complications, and survival among older women with invasive breast cancer. JAMA (2012) 307:1827-37. doi:10.1001/jama.2012.3481

Conflict of Interest Statement: The authors declare that the research was conducted in the absence of any commercial or financial relationships that could be construed as a potential conflict of interest.

Received: 09 July 2014; accepted: 30 September 2014; published online: 22 October 2014.

Citation: Merino Lara TR, Fleury E, Mashouf S, Helou J, McCann C, Ruschin M, Kim A, Makhani N, Ravi A and Pignol J-P (2014) Measurement of mean cardiac dose for various breast irradiation techniques and corresponding risk of major cardiovascular event. Front. Oncol. 4:284. doi: 10.3389/fonc.2014.00284

This article was submitted to Radiation Oncology, a section of the journal Frontiers in Oncology.

Copyright (c) 2014 Merino Lara, Fleury, Mashouf, Helou, McCann, Ruschin, Kim, Makhani, Ravi and Pignol. This is an open-access article distributed under the terms of the Creative Commons Attribution License (CC BY). The use, distribution or reproduction in other forums is permitted, provided the original author(s) or licensor are credited and that the original publication in this journal is cited, in accordance with accepted academic practice. No use, distribution or reproduction is permitted which does not comply with these terms. 